\title{
EVRIMSEL OVUN OLARAK KURUMSAL KONTROL ETKILEŞIMI
}

\author{
Yru. Doç. Dr. Uğur Soytas \\ Ortadoğu Teknik Üniversitesi \\ iktisadi ve idari Bilimler Fakültesi
}

\section{Özet}

Yatırımcı ve hedef yönetim arasındaki kurumsal kontrol etkileşimini modellemek için oyun teorisi uygun bir araçtır. Ancak geleneksel oyun teorisi araçlan genelde birden fazla Nash dengesini işaret etmektedir. Bu makalede Mason ve digerlerinde (2003) yer alan asimetrik bilgili etap oyunu, evrimsel ve dinamik bir kurumsal kontrol oyunu olarak modellenmiştir. Geniş denge kümeleri yerine, evrimsel oyunun çözumünde olası kararlı durum olabilecek sekiz vaka bulunmuştur. Vakalardaki seçimlerin evrimsel kararlı stratejiler olabilmeleri için gerekli olan kazanç yapılan ortaya konmuştur. Böylece popillasyondaki yatırımcı tipi olasılık dağlım tahmin edildiģinde, oyunun tek kararlı durumunu bulmak mümkün olmaktadır. Bu denge oyuncuların rasyonel olduğu varsayımına dayanmamaktadır. oyunu, Mason.

Anahtar Kelimeler: Kurumsal kontrol, evrimsel kararlı stratejiler, oyun teorisi, asimetrik bilgili etap

\section{Corporate Control Interaction as an Evolutionary Game}

\section{Abstract}

Game theory is an appropriate tool to model the interaction between investor and management in a corporate control interaction. However, traditional game theoretic tools generally yield multiple equilibria. In this paper we take the assymmetric information stage game in Mason et al. (2003) and model it as an evolutionary corporate control game. Instead of wide sets of multiple equilibria, we point out eight candidates for the stable state of the population. We also show the conditions for the choices under each case to be evolutionarily stable strategies. Hence, when the probability distribution of investor types is estimated it is possible to identify a unique equilibrium state of the game. This equilibrium does not depend on the rationality assumption.

Keywords: Corporate control, evolutionarily stable strategies, game theory, assymmetric information stage game, Mason. 


\section{Evrimsel Oyun Olarak Kurumsal Kontrol Etkileşimi}

\section{Giriş}

Kurum yöneticilerinin büyük hissedarların zoraki el koyma karan ile karşı karşıya kaldıklanında neden ve ne tür tepkiler verdiklerini konu edinen hem ampirik hem de kuramsal çok geniş bir yazın vardır (kapsamlı yazın taraması için bakınız Dahya / Mcconnell, 2005; Hermalin / Weisback, 2003). Rasyonel bir yönetici zoraki satın alma durumuyla karşılaşığında, kurumun kontrol devrinden sonraki getiri akımı ile mevcut durumun devamında oluşacak getiri akımını karşılaştırarak karşı koyma ile işbirliği yapma arasında bir seçim yapacaktır (Noe / Pi, 2000). Yöneticiler hem gelecek hem de yatınmcı hakkında tam bilgiye sahipken bu tip kararlan vermek sadece çok basit bir işlem gerektirmektedir. Ancak, kurum yönetimi hiçbir zaman gelecekteki getiri akımlan ya da yatınmcının şirkete değer kazandını kazandıramayacağı konusunda tam ve kesin bilgiye sahip olmamaktadırlar. Kurumsal kontrol yazınının bir bölümü, yatırımcıların yöneticiler tarafından nasıl algılandığının yönetimin alacağı tavır üzerindeki etkisini incelemektedir. Ne ampirik ne de teorik yazında tam anlamıla uzlaşmaya vanılmamıştır. Yani bazı büyük hissedarlann zorla el koyma yoluna giderken, diğerlerinin tepkisiz veya pasif kaldıklanı, buna cevaben bazı yöneticilerin karşı koymayı, bazılanının ise işbirliği yoluna gimeyi seçtikleri ortaya konmuştur (Mason vd., 2003).

Bulgular ne kadar karışık da olsa, yatırnmcı ve kurum yönetimi arasındaki çıkar çatışmasının aracılık kuramı kapsamına girdiği açıkır. Son zamanlarda oyun kuramı kurumsal kontrol alanında stratejik çıkar çatışmalarını modellemeye yarayan standart bir araç olmuştur (Cyert vd., 2002; Mason vd., 2003; Hu / Shapley, 2003). Geleneksel oyun kuramı araçlannın kullanıldığı bu çalışmaların en büyük problemi ise, birden fazla Nash dengesinin ${ }^{1}$

1 Nash dengesinin matematiksel olmayan tanımı şöyle özetlenebilir: Bu dengede diğer oyuncuların stratejileri sabitken, hiçbir oyuncu stratejisini değiştirerek kazancımı yükseltemez. 
bulunmasıdır. Bu Nash dengelerinin kapsamı ampirik ve teorik yazında yer alan her türlü sonucu içerecek kadar geniştir. Dolayısıyla oyunun hangi dengeye yakınsayacağını tahmin etmek güçtür.

Bu yazının karşılaştığı başka iki sorun ise yine geleneksel oyun kuramının yükünü çeken Nash dengesinin kullanımından kaynaklanmaktadır. Birincisi, Nash dengesi kendine zorlayan bir kavramdır ve dengeye nasil ulaşıldığını açıklamaz (Soytaş, 2002; Soytaş / Becker, 2003; Soytaş, 2005). İkincisi, Nash dengesi sadece oyuncuların rasyonel olduğu varsayımına değil, rasyonelliğin ortak bilgi ${ }^{2}$ olduğu varsayımına da dayanmaktadır (Dixit / Skeath, 1999: 216). Oysa, gerçek hayatta, yatınmcıların verdikleri sinyallere bakarak eksik bilgi ile karar verecek olan yöneticiler, seçtikleri stratejilerden hangisinin başanlı hangisinin başarısız olduğunu gözlemleyerek ögrenebilmektedirler. Kabaca, yöneticiler başarılı olduğunu gözlemledikleri stratejileri taklit edecek, başansız görünenleri ise kullanmayacaktır. Dolayısıyla, bu deneme, yanılma, öğrenme sürecinde sürekli başansız olan bazı stratejilerin yavaş yavaş elenecekleri söylenebilir. Bu sürecin sonsuza dek tekrarlandığı düşünülürse, Nash dengesine göre rasyonel olmayan davranışlann neden gerçek hayatta karşımıza çıktıkları da anlaşılabilir. Doğal seçim analojisini andıran bu dinamiği modellemek için geleneksel oyun kuramı araçları yetersiz kalmaktadır (Samuelson, 1997).

Bu makalede, yatırımcı tipinin, zorla elde etme tehtidi ile karşı karşıya kalan kurum yöneticilerinin strateji seçimlerine etkisi simetrik bilgi yapısına sahip dinamik bir oyun olarak modellenmiş ve evrimsel oyun kuramı araçları ile çözümlenmiştir. Evrimsel oyunun etap oyunu ve kazanç fonksiyonlan, geleneksel oyun teoretik modelleme yapılmış olan Mason ve diğerlerinden (2003) alınmıştır. Orijinal oyunun aksine, sadece oyunun kazanç yapısının sınırlandırdığ koşullar altında hangi stratejilerin replikatör dinamiğinden sağ çıkıkları ortaya konmuştur. Dolayısıyla, genel bir koşul altında yatınmo ve yönetici stratejileri popülasyonunun alcağı tek bir kararlı nokta ortaya çıkarılarak birden fazla denge problemi aşılmıştır. Böylelikle yatırımcı tipi olasılık dağılımı tahmin edilerek oyunun sonucunun hangi tek kararlı dengeye yakınsayacağı bulunabilecektir. Ayrıca, bu sonuç rasyonelliğin ortak bilgi olduğu, hattâ oyuncuların rasyonel oldukları varsayımına da dayanmamaktadır.

2 Rasyonelliğin ortak bilgi olması sonsuza uzanan bir mantık zinciri gerektirir: Birinci oyuncu ikinci oyuncunun rasyonel olduğunu biliyor; ikinci oyuncu birincinin rasyonel olduğunu biliyor; birinci oyuncu ikinci oyuncunun birincinin rasyonel olduğunu biliyor vs. 


\section{Asimetrik Bilgili Dinamik Etap Oyunu}

Bu etap oyunu Mason ve diğerleri (2003) tarafından incelenen tekrar edilen oyundan alınmıştır. İki oyunculu dinamik oyunda birinci oyuncu yatınmcı $(\mathrm{Y})$ ve ikinci oyuncu hedef şirketin yönetimidir $(\mathrm{H})$. Birinci oyuncu popülasyonu iki tip yatınmcıdan oluşmaktadır, Güçlü $(G)$ ve Zayıf $(Z)$. Bu tiplerin popülasyondaki göreceli frekanslan ise sırasıyla $\delta_{G}$ ve $\left(1-\delta_{G}\right)$ ile gösterilmiştir. Mücadelenin başında yatınmcı kendi tipini bilmekte fakat hedef yönetim sadece yatınmcının hangi olasılıkla $G\left(\delta_{G}\right)$ ve hangi olasılıkla $Z$ ( 1 $\delta_{G}$ ) olduğunu bilmektedir. Bir başka deyişle oyundaki ilk hareket yatınmcının tipini olsalık dağllımına göre rastgele belirleyen doğaya aittir. Etap oyunu Çizim 1'de gösterilmektedir.

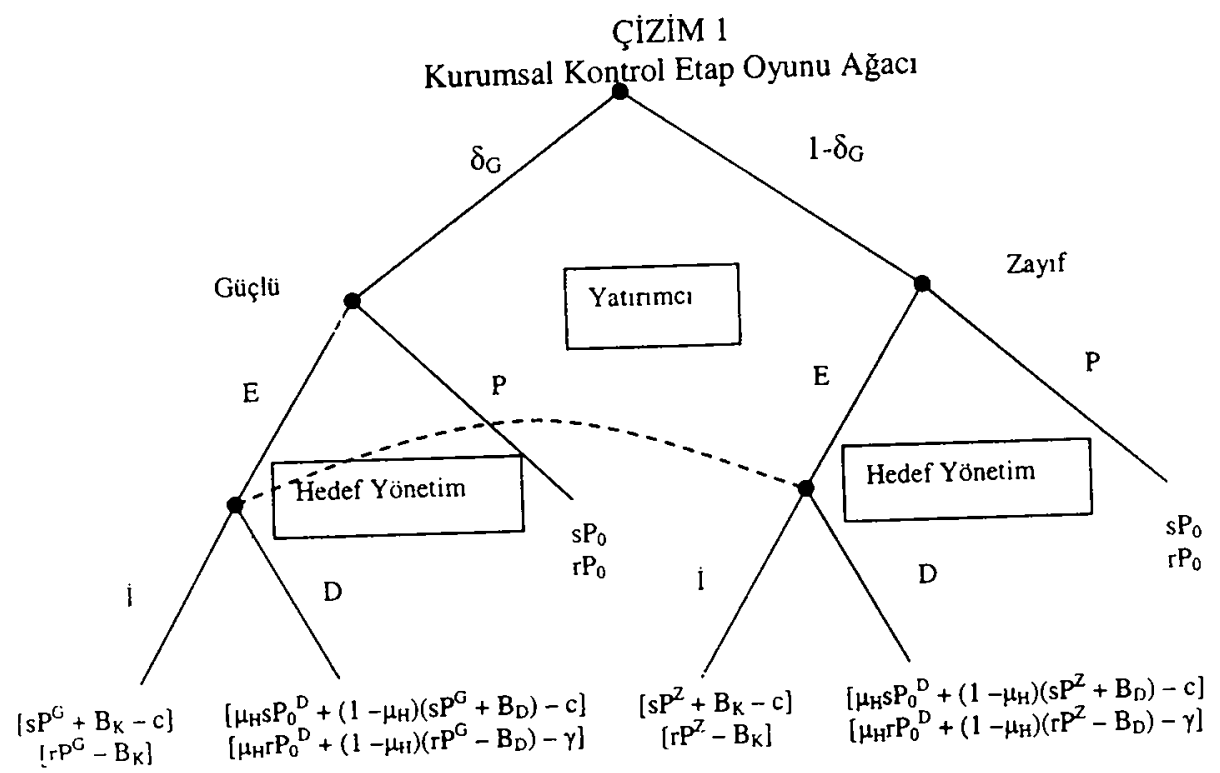

Etap oyunu iki safhada gerçekleşmektedir. Illk safhada yatınmcı sadece kendi bilgi kümesinde yer alan tipine göre bir strateji seçer. Yatırımcının strateji kümesi, $S_{Y}=\{E, P\}$, iki stratejiden oluşmaktadır: $E$ zorla elde etmeye çalışmak ve $P$ pasif kalmak. İkinci safhada ise sıra yönetime gelmektedir. Yönetim, ilk safhada yatırımcının seçimini görmekte ancak yatırımcının tipinden emin olamamaktadır. Hedef yönetimin aksiyon kümesinde ise, $A_{H}=$ 
bulunmaktadır ${ }^{3}$. Direnmenin maliyeti $\gamma$, başan olasılığı ise $\mu_{\mathrm{H}}$ ile gösterilmektedir.

Çizim 1'de oyun ağacının başlangı̧ noktası tabiat tarafından gerçekleştirildiği varsayılan bir aksiyonu belirtmektedir. Bu önemsiz (trivial) hareket yatırımcı popülasyonundaki tiplerin olasılık dağılımını belirler. Aynca, ilk hareketi gerçekleştiren yatırımcının kendi tipinin ne olduğunu bildiği açıktır. Olasılık dağılımı belirlendikten sonra, oyun ağacında yatınmcının strateji seçenekleri E ve P dallan ile gösterilmektedir. İkinci safhada hedef yönetimin karar noktalarını birbirine bağlayan kesik çizgiler ise oyunun asimetik bilgi niteliğini yansıtmaktadır. Yani sıra ikinci oyuncuya geldiğinde bu oyuncu hangi karar noktasında (solda m, sağda mu) olduğunu (yatırımcının tipini) kesin olarak bilmemektedir. Oyun ağacının bitiş noktalarına ise ilk satırda birinci oyuncunun, ikinci satırda ise ikinci oyuncunun kazançlan yerleştirilmiştir. Çizimden de anlaşılacağı gibi, yatınmcı ve hedef yönetimin olası her strateji kombinasyonu için tanımlı bir kazanç profili vardır.

\section{Evrimsel Oyunun Tamamı}

Mason ve diğerleri (2003) etap oyununu tekrar edilen bir oyunun parçası olarak nitelerken, bu makalede aynı etap oyunu evrimsel bir oyunun parçası olarak ele alınmıştır. Tabiat, belirledigi olasılıklara göre, yatırımcı ve hedef yönetim popülasyonlanndan birer oyuncuyu etap oyununu oynamak üzere rastgele seçer. Bu rastgele eşleşmeler sonsuz kez tekrarlanır. Oyuncu popülasyonundaki herkes etap oyunlannda hangi stratejilerin seçildiğini ve sonuçta hangi kazançlann elde edildiğini görür. Bir oyuncu kendi stratejisini ancak ve ancak gözlemlenen strateji daha yüksek kazanç sağlıyorsa değiştirir. Böylece daha çok rastgele eşleşme yapıldıkça, averajın üstünde kazanç saplayan stratejileri seçen oyuncuların oranı artacak, başarısız staretjileri seçenlerin oranı ise düşecektir.

$G$ ve $Z$ tipi yatınmcılar arasından $E$ stratejisi seçimiyle başlayanların oranlan sirasiyla $p_{1}$ ve $p_{2}$ olsun. Bu durumda $G$ ve $Z$ tipi yatırımcilardan $P$ seçimiyle popülasyonda yer alanlan oranları $\left(1-p_{1}\right)$ ve $\left(1-p_{2}\right)$ olacaktır. Ayrıca, yine sırasıyla, $\mathrm{p}_{3}$ I seçimiyle ve $\left(1-\mathrm{p}_{3}\right)$ de $\mathrm{D}$ seçimiyle başlayan ikinci oyuncularn oranlan olsun. Bu oranlara göre herhangi bir anda oyuncu

3 İkinci oyuncu olan hedef yönetim, stratejisini ilk safhadaki rakip oyuncunun seçimine koşullandırabileceği için iki aksiyon alternatifine ama aslında dört stratejiye sahiptir. Strateji tam bir aksiyon planıdır. Örneğin, "yatırımcı E'yi seçerse diren, P'yi seçerse direnme" tek bir stratejidir. 
popülasyonunun durumu $\mathrm{p}=\left\{\left(\mathrm{p}_{1}, 1-\mathrm{p}_{1}\right),\left(\mathrm{p}_{2}, 1-\mathrm{p}_{2}\right),\left(\mathrm{p}_{3}, 1-\mathrm{p}_{3}\right)\right\}$ olarak gösterilebilir.

\section{Kazançlar}

Mason ve diğerleri (2003) tarafından belirlenen kazanç fonksiyonlarına göre $\mathrm{P}_{0}$ bir hissenin oyun başlamadan önceki fiyatını, $\mathrm{P}_{0}{ }^{\mathrm{D}}$ hissenin başanılı bir direnis sonrası fiyatını, $P^{G}$ ve $P^{Z}$ ise sırasıyla güçlü ve zayıf yatırımcının kontrolündeki fiyatını göstermektedir. $\mathrm{P}^{\mathrm{G}}$ ve $\mathrm{P}^{Z}$ yönetimin zorla elde etmeye direnip direnmemesinden bağımsız oldugu varsayılmaktadır. Fiyatların büyüklükleri $\mathrm{P}^{\mathrm{G}}>\mathrm{P}_{0}>\mathrm{P}_{0}{ }^{\mathrm{D}}>\mathrm{P}^{Z}$ olarak sıralanmaktadır. Zorla elde etme stratejisinin maliyeti yatınmcı tipi ne olursa olsun c olarak ele alınmıştır.

$B_{K}$ kontrolden kaynaklanan getirilerin, $B_{D}$ ise yönetimin direndiği ancak zorla elde etmenin başarılı olduğu zaman yönetimden yatınmcıya geçen getirilerin bugünkui değerini göstermektedir. Ayrıca kazançlar yönetimi direnip başarılı olmamaktan ziyade işbirliği yapmayı tercih etmeye yönlendirmektedir, yani $B_{D}>B_{K}$. Ayrıca $s$ ve $r$ sırasıyla yatırımcı ve yönetimin toplam özsermaye paylarını göstermektedir. Bir başka varsayım ise $B_{D}>P^{G}-P_{0}^{D}$ ve $B_{D}+P^{Z}>$ $\mathrm{P}_{0}{ }^{\mathrm{D}}$ eşitsizlikleri ile belirtilmiştir. İlk eşitsizlik yatırımcı mücadeleyi kazandığı zaman yönetimin kaybının, fiyattaki potansiyel artıştan yüksek olduğunu, ikincisi ise tipi ne olursa olsun yatırımcının zorla elde etmede başarılı olmayı tercih ettiğini göstermektedir.

Çizim 1'deki etap oyunundan da anlaşılacağı gibi, ilk safhada yatırımcı pasif kalmayı $(\mathrm{P})$ seçerse, oyun sona ermekte ve hissenin değeri $\mathrm{P}_{0}$ olarak kalmaktadır. Böylece yatırımcı $\mathrm{sP}_{0}$, yönetici ise $\mathrm{rP}_{0}$ kazanç elde etmektedir. Eğer $G$ tipinde bir yatırımcı işbirliği yapan bir yönetim ile eşleşirse kazançlanı sırasıyla $\left[s \mathrm{P}^{\mathrm{G}}+\mathrm{B}_{\mathrm{K}}-\mathrm{c}\right]$ ve $\left[\mathrm{rP}^{\mathrm{G}}-\mathrm{B}_{\mathrm{K}}\right]$ olarak gösterilebilir. Zayıf bir yatırımcı işbirlikçi yönetimin bulunduğu şirkette saldında bulunursa, yatırımcı $\left[\mathrm{sP}^{2}+\mathrm{B}_{\mathrm{K}}\right.$ $-c]$ ve yönetim $\left[\mathrm{rP}^{Z}-\mathrm{B}_{K}\right]$ kazanir.

Eğer ikinci oyuncu direnen bir yönetim olursa iki oyuncunun kazançları da direnişin başarılı olma olasılığına $\left(\mu_{\mathrm{H}}\right)$ bağlıdır. Bu durumda gưçlü birinci oyuncu saldırdığında yatırımcı $\left[\mu_{H} \mathrm{sP}_{0}{ }^{D}+\left(1-\mu_{H}\right)\left(s \mathrm{P}^{\mathrm{C}}+\mathrm{B}_{\mathrm{D}}\right)-\mathrm{c}\right]$, yönetim ise $\left[\mu_{H} \mathrm{rP}_{0}{ }^{\mathrm{D}}+\left(1-\mu_{\mathrm{H}}\right)\left(\mathrm{rP}^{\mathrm{G}}-\mathrm{B}_{\mathrm{D}}\right)-\gamma\right]$ beklenen getirilere sahip olur. Eğer zorla elde etmeye yönelen zayıf yatırımcı ise yatırımcı ve direniş̧̧i yönetim kazançlan sirasiyla $\left[\mu_{\mathrm{H}} \mathrm{sP}_{0}{ }^{\mathrm{D}}+\left(1-\mu_{\mathrm{H}}\right)\left(s \mathrm{P}^{\mathrm{Z}}+\mathrm{B}_{\mathrm{D}}\right)-\mathrm{c}\right]$ ve $\left[\mu_{\mathrm{H}} \mathrm{r} \mathrm{P}_{0}{ }^{\mathrm{D}}+\left(1-\mu_{\mathrm{H}}\right)\left(\mathrm{rP}^{2}-\mathrm{B}_{\mathrm{D}}\right)-\gamma\right]$ olur. 


\section{Varsayımlar}

$\mathrm{Bu}$ evrimsel oyunun kurgusunda ve kazançlanında açıkça belirtilen varsayımlar dışında, arka planda kalan üç çok önemli varsayım daha vardır. Bunlardan birincisi tüm aksiyon alternatifleri (E, P, D, I) popülasyonda sıfırın üstünde bir olasılıkla yer almaktadırlar. Zorla elde etme yazınında yer alan çelişkili bulgular ışı̆̆ında bu çok gerçek dışı bir varsayım olarak göze batmamaktadır ve oyuncu popülasyonunun zengin olmasını garanti etmektedir. İkinci varsayım ise oyuncuların davranışlanını (aksiyon seçimlerini) sadece taklit yoluyla değiştirdikleridir. Taklit en basit öğrenme modeli olarak kullanılan bir varsayımdır (Schlag, 1997). Ayrıca rasyonellik ve rayonelliğin ortak bilgi olması varsayımlanın gerektirmez. Son olarak, çok bağlayıcı olmasa da oyunculann miyopik olduklan varsayımıdır. Yani hiçbir oyuncu gelecekte oyuncu popülasyonunun kararlı durumunun ne olacağını kestiremez. Bu da aslında evrimsel oyunu gerçekliğe daha çok yaklaştıran bir varsayım olarak ele alınabilir, çünkü Nash dengesi gibi oyunculann mükemmel hesap yapabilen, hem geçmişi unutmayan, hem de gelecek hakkında rasyonel projeksiyonlar yapabilen oyuncular gerektirmez.

\section{Oyunun Çözümlenmesi}

Samuelson 'a (1997) göre bir popülasyonun kararlı durumları değişik dinamiklerin kullanılmasına göreceli olarak tepkisiz kalmaktadır. Bununla birlikte taklitin önemli rol oynadığı öğrenme modelleri replikatör dinamiklerine yol açabilir. Schlag (1997) da aslında bu tip modellerin limitte replikatör dinamiklerine yol açtığını göstermiştir. Bu bölümde replikatör dinamiklerinin (Taylor / Jonker, 1978) uygulaması yapılacaktır.

Replikatör denklemi aşağıdaki gibidir:

$d \mathrm{p}_{\mathrm{i}} / d \mathrm{t}=\left[\pi\left(\mathrm{a}_{\mathrm{i}}, \mathrm{p}\right)-\pi\left(\mathrm{p}_{\mathrm{i}}, \mathrm{p}\right)\right] \mathrm{p}_{\mathrm{i}}$

Bu denklemde $a_{i} \in A_{j}, \pi\left(a_{i}, p\right)$ i oyuncusunun popülasyondaki $p=\left\{\left(p_{1}, 1\right.\right.$ $\left.\left.-p_{1}\right),\left(p_{2}, 1-p_{2}\right),\left(p_{3}, 1-p_{3}\right)\right\}$ dağılımına karşı $a_{i}$ oynadığı zamanki kazancı $(i=$ $1,2,3$ ve $j=Y, H)$, ve $\pi\left(p_{i}, p\right) p_{i}$ aksiyon dağılımının $p$ popülasyon dağılıms karşısındaki kazancı, yani popülasyonda yer alan i tipli oyuncunun averaj getirisi olmaktadir.

Buna göre hem güçlü hem de zayıf yatımcılann E (zorla elde etme) ile hedef yönetimin İ (işbirliği) saf stratejilerini ele alacak olursak, popülasyon dağılımına karşı getirileri aşağıdaki gibi gösterebiliriz:

i) $\pi_{\mathrm{G}}(\mathrm{E}, \mathrm{p})=\mathrm{p}_{3}\left[s \mathrm{P}^{\mathrm{G}}+\mathrm{B}_{\mathrm{K}}-\mathrm{c}\right]+\left(1-\mathrm{p}_{3}\right)\left[\mu_{\mathrm{H}} \mathrm{s} \mathrm{P}_{0}{ }^{\mathrm{D}}+\left(1-\mu_{\mathrm{H}}\right)\left(s \mathrm{P}^{\mathrm{G}}+\mathrm{B}_{\mathrm{D}}\right)-\mathrm{c}\right]$

ii) $\pi_{Z}(E, p)=p_{3}\left[s P^{Z}+B_{K}-c\right]+\left(1-p_{3}\right)\left[\mu_{H} s P_{0}{ }^{D}+\left(1-\mu_{H}\right)\left(s P^{Z}+B_{D}\right)-c\right]$ 
iii) $\pi_{\mathrm{H}}(\dot{\mathrm{I}}, \mathrm{p})=\delta_{\mathrm{G}}\left(\mathrm{p}_{1}\left[\mathrm{rP}^{\mathrm{G}}-\mathrm{B}_{\mathrm{K}}\right]+\left(1-\mathrm{p}_{1}\right) \mathrm{rP}_{0}\right)+\left(1-\delta_{\mathrm{G}}\right)\left(\mathrm{p}_{2}\left[\mathrm{sP}^{Z}+\mathrm{B}_{\mathrm{K}}-\right.\right.$ c] $\left.+\left(1-\mathrm{p}_{2}\right) \mathrm{rP}_{0}\right)$.

$\pi_{G}, \pi_{Z}$, ve $\pi_{H}$ sırasıyla güçlü yatırımcı, zayıf yatırımcı ve hedef yönetim kazanç fonksiyonlandır. Tüm oyuncu tiplerinin $(i=1,2,3)$ averaj kazançları hesaba katıldığında repliklatör dinamikleri şöyle oluşur:

$d \mathrm{p}_{1} / d \mathrm{t}=\mathrm{p}_{1}\left(1-\mathrm{p}_{1}\right)\left[\left(\mathrm{p}_{3}\left(\mathrm{sP}^{\mathrm{G}}+\mathrm{B}_{\mathrm{K}}-\mathrm{c}\right)+\left(1-\mathrm{p}_{3}\right)\left[\mu_{\mathrm{H}} \mathrm{s} \mathrm{P}_{0}{ }^{\mathrm{D}}+\left(1-\mu_{\mathrm{H}}\right)\left(\mathrm{sP}^{\mathrm{G}}+\right.\right.\right.\right.$ $\left.\left.\left.\left.\mathrm{B}_{\mathrm{D}}\right)-\mathrm{c}\right]\right)-\left(\mathrm{p}_{3}\left[\mu_{\mathrm{H}} \mathrm{rP}_{0}{ }^{\mathrm{D}}+\left(1-\mu_{\mathrm{H}}\right)\left(\mathrm{rP}^{\mathrm{C}}-\mathrm{B}_{\mathrm{D}}\right)-\gamma\right]+\left(1-\mathrm{p}_{3}\right) \mathrm{rP}_{0}\right)\right]$

$d \mathrm{p}_{2} / d \mathrm{t}=\mathrm{p}_{2}\left(1-\mathrm{p}_{2}\right)\left[\left(\mathrm{p}_{3}\left(\mathrm{sP}^{Z}+\mathrm{B}_{\mathrm{K}}-\mathrm{c}\right)+\left(1-\mathrm{p}_{3}\right)\left[\mu_{\mathrm{H}} \mathrm{s} \mathrm{P}_{0}^{\mathrm{D}}+\left(1-\mu_{\mathrm{H}}\right)\left(\mathrm{sP}^{Z}+\right.\right.\right.\right.$ $\left.\left.\left.\left.B_{D}\right)-c\right]\right)-\left(p_{3}\left[\mu_{H} r P_{0}^{D}+\left(1-\mu_{H}\right)\left(r P^{2}-B_{D}\right)-\gamma\right]+\left(1-p_{3}\right) r P_{0}\right)\right]$

$d \mathrm{p}_{3} / d \mathrm{t}=\mathrm{p}_{3}\left(1-\mathrm{p}_{3}\right)\left[\mathrm{P}_{\mathrm{L}}\left(\mathrm{p}_{1}\left(\mathrm{rP}^{\mathrm{G}}-\mathrm{B}_{\mathrm{K}}\right)+\left(1-\mathrm{p}_{1}\right) \mathrm{sP}_{0}\right)+\left(1-\mathrm{P}_{\mathrm{L}}\right)\left(\mathrm{p}_{2}\left(\mathrm{rP}^{2}-\right.\right.\right.$ $\left.\left.\left.\mathrm{B}_{\mathrm{K}}\right)+\left(1-\mathrm{p}_{2}\right) \mathrm{sP}_{0}\right)\right]$.

Gardner ve Morris'i (1991) takip ederek bu denklem sistemini aşağıdaki gibi yazabiliriz:

$$
\frac{\partial \mathrm{p}_{\mathrm{i}}}{\partial \mathrm{t}}=\mathrm{p}_{\mathrm{i}}\left(1-\mathrm{p}_{\mathrm{i}}\right) \mathrm{F}_{\mathrm{i}}(\mathrm{p})
$$

Burada $i=1,2,3$ ve

$F_{1}(p)=\left[\left(p_{3}\left[s P^{G}+B_{K}-c\right]+\left(1-p_{3}\right)\left[\mu_{H} s P_{0}^{D}+\left(1-\mu_{H}\right)\left(s P^{G}+B_{D}\right)-c\right]\right)-\right.$ $\left.\left(\mathrm{p}_{3}\left[\mu_{\mathrm{H}} \mathrm{r} \mathrm{P}_{0}{ }^{\mathrm{D}}+\left(1-\mu_{\mathrm{H}}\right)\left(\mathrm{rP}^{\mathrm{G}}-\mathrm{B}_{\mathrm{D}}\right)-\gamma\right]+\left(1-\mathrm{p}_{3}\right) \mathrm{rP}_{0}\right)\right]$

$F_{2}(p)=\left[\left(p_{3}\left[s P^{Z}+B_{K}-c\right]+\left(1-p_{3}\right)\left[\mu_{H} s P_{0}^{D}+\left(1-\mu_{M}\right)\left(s P^{Z}+B_{D}\right)-c\right]\right)-\right.$ $\left.\left(\mathrm{p}_{3}\left[\mu_{\mathrm{H}} \mathrm{r} \mathrm{P}_{0}{ }^{\mathrm{D}}+\left(1-\mu_{\mathrm{H}}\right)\left(\mathrm{rP}^{\mathrm{Z}}-\mathrm{B}_{\mathrm{D}}\right)-\gamma\right]+\left(1-\mathrm{p}_{3}\right) \mathrm{rP}_{0}\right)\right]$ $\left.\left.\left.\mathrm{p}_{2}\right) \mathrm{sP}_{0}\right)\right]$.

$\mathrm{F}_{3}(\mathrm{p})=\left[\mathrm{P}_{\mathrm{L}}\left(\mathrm{p}_{1}\left[\mathrm{rP}^{\mathrm{G}}-\mathrm{B}_{\mathrm{K}}\right]+\left(1-\mathrm{p}_{1}\right) \mathrm{sP}_{0}\right)+\left(1-\mathrm{P}_{\mathrm{L}}\right)\left(\mathrm{p}_{2}\left[\mathrm{rP}^{Z}-\mathrm{B}_{\mathrm{K}}\right]+(1-\right.\right.$

Dolayısıyla $F_{i}(p)$ pozitifken $i$ oyuncusu $(i=1,2,3)$ popülasyon averajının üzerinde kazanç elde etmektedir ve alt popülasyonda yer alan diğer oyuncular onun stratejisini taklit etmek istemektedir. Böylece i tipinin popülasyonaki oranı artacaktır. Negatif $F_{i}(p)$ 'ye sahip bir tipin ise oranı düşecek ve replikatör dinamiğinden sağ çıkamayacaktır.

Yukandaki otonom diferansiyel denklemler sisteminin evrimsel kararl stratejileri ${ }^{4}(E K S) \mathrm{p}^{*}=\left\{\left(\mathrm{p}_{1}{ }^{*}, 1-\mathrm{p}_{1}{ }^{*}\right),\left(\mathrm{p}_{2}{ }^{*}, 1-\mathrm{p}_{2}{ }^{*}\right),\left(\mathrm{p}_{3}{ }^{*}, 1-\mathrm{p}_{3}{ }^{*}\right)\right\}$ ise $\mathrm{p}^{*}$ dinamik kararl system dengesini niteler (Gardner / Morris, 1991). Selten (1980) karma stratejilerin asimetrik oyunlarda EKS olamayacağını göstermiştir. Dolayısıyla, EKS'yi birim küpün köşelerinde aramak gerekir. Liapunov teoremine göre denge noktası olan $\mathrm{p}^{*}$, Jacobian matrisin $\mathrm{p}^{*}$ noktasında

4 Evrimsel kararı ı stratejiler (Evolutionary stable strategies) tanımı için bakınız Soytaş (2002). 
değerlendirilen tüm karakteristik kökleri negatif ise, asimptotik olarak karalıdır (Gandolfo, 1996). Aynı koşullar EKS için de geçerlidir, çünkü asimetrik mücadelelerde tam Nash dengesi, EKS ve dinamik kararlılı birbirini ima eder.

Bu bilgiler ışığında evrimsel kurumsal kontrol oyununa baktığımızda tam sekiz adet olası kararlı nokta ya da potansiyel EKS görürüz. Karakteristik kökler $\mathrm{p}^{*}$ noktasında irdelendiğinde bu sekiz adayın her birisinin EKS olabilmesi için gerekli koşulları elde ederiz. Bu değerlndirmelere göre popülasyonun 8 adet potansiyel kararlı durumunun her birinin gerçekleşebilmesi için gereken kazanç yapısı aşağıda verilmiştir:

Vaka $1 . \mathrm{p}^{*}=\{(1,0),(1,0),(1,0)\}$ EKS ise:

1. $\left[s \mathrm{P}^{\mathrm{G}}+\mathrm{B}_{\mathrm{K}}-\mathrm{c}\right]>\left[\mu_{\mathrm{H}} \mathrm{P}_{0}{ }^{\mathrm{D}}+\left(1-\mu_{\mathrm{H}}\right)\left(\mathrm{rP}^{\mathrm{G}}-\mathrm{B}_{\mathrm{D}}\right)-\gamma\right]$

2. $\left[s \mathrm{P}^{Z}+\mathrm{B}_{\mathrm{K}}-\mathrm{c}\right]>\left[\mu_{\mathrm{H}} \mathrm{P}_{0}{ }^{\mathrm{D}}+\left(1-\mu_{\mathrm{H}}\right)\left(\mathrm{rP}^{\mathrm{Z}}-\mathrm{B}_{\mathrm{D}}\right)-\gamma\right]$

3. $\delta_{\mathrm{G}}\left[\mathrm{rP}^{\mathrm{G}}-\mathrm{B}_{\mathrm{K}}\right]+\left(1-\delta_{\mathrm{G}}\right)\left[\mathrm{rP}^{\mathrm{Z}}-\mathrm{B}_{\mathrm{K}}\right]>0$.

Vaka 2. $\mathrm{p}^{*}=\{(1,0),(1,0),(0,1)\}$ EKS ise:

1. $\left[\mu_{\mathrm{H}} \mathrm{SP}_{0}^{\mathrm{D}}+\left(1-\mu_{\mathrm{H}}\right)\left(\mathrm{sP} \mathrm{P}^{\mathrm{G}}+\mathrm{B}_{\mathrm{D}}\right)-\mathrm{c}\right]>\mathrm{rP}_{0}$

2. $\left[\mu_{H} S P_{0}^{D}+\left(1-\mu_{H}\right)\left(s P^{Z}+B_{D}\right)-c\right]>\mathrm{rP}_{0}$

3. $\delta_{\mathrm{G}}\left[\mathrm{rP}^{\mathrm{G}}-\mathrm{B}_{\mathrm{K}}\right]+\left(1-\delta_{\mathrm{G}}\right)\left[\mathrm{rP}^{\mathrm{Z}}-\mathrm{B}_{\mathrm{K}}\right]>0$.

Vaka 3. $\mathrm{p}^{*}=\{(1,0),(0,1),(1,0)\}$ EKS ise:

1. $\left[s \mathrm{P}^{\mathrm{G}}+\mathrm{B}_{\mathrm{K}}-\mathrm{c}\right]>\left[\mu_{\mathrm{H}} \mathrm{PP}_{0}^{\mathrm{D}}+\left(1-\mu_{\mathrm{H}}\right)\left(\mathrm{rP}^{\mathrm{Z}}-\mathrm{B}_{\mathrm{D}}\right)-\gamma\right]$

2. $\left[\mu_{\mathrm{H}} \mathrm{rP}_{0}{ }^{\mathrm{D}}+\left(1-\mu_{\mathrm{H}}\right)\left(\mathrm{rP}^{\mathrm{Z}}-\mathrm{B}_{\mathrm{D}}\right)-\gamma\right]>\left[\mathrm{sP}^{\mathrm{Z}}+\mathrm{B}_{\mathrm{K}}-\mathrm{c}\right]$

3. $\delta_{\mathrm{G}}\left[\mathrm{rP}^{\mathrm{G}}-\mathrm{B}_{\mathrm{K}}\right]+\left(1-\delta_{\mathrm{G}}\right) \mathrm{sP}_{0}>0$.

Vaka 4. $\mathrm{p}^{*}=\{(0,1),(1,0),(1,0)\}$ EKS ise:

1. $\left[\mu_{\mathrm{H}} \mathrm{rP}_{0}^{\mathrm{D}}+\left(1-\mu_{\mathrm{H}}\right)\left(\mathrm{rP}^{\mathrm{Z}}-\mathrm{B}_{\mathrm{D}}\right)-\gamma\right]>\left[\mathrm{sP}^{\mathrm{G}}+\mathrm{B}_{\mathrm{K}}-\mathrm{c}\right]$

2. $\left[\mathrm{sP}^{2}+\mathrm{B}_{\mathrm{K}}-\mathrm{c}\right]>\left[\mu_{\mathrm{H}} \mathrm{rP}_{0}{ }^{\mathrm{D}}+\left(1-\mu_{\mathrm{H}}\right)\left(\mathrm{rP}^{2}-\mathrm{B}_{\mathrm{D}}\right)-\gamma\right]$

3. $\delta_{\mathrm{G}} \mathrm{s} \mathrm{P}_{0}+\left(1-\delta_{\mathrm{G}}\right)\left[\mathrm{rP}^{\mathrm{Z}}-\mathrm{B}_{\mathrm{K}}\right]>0$.

Vaka 5. $\mathrm{p}^{*}=\{(1,0),(0,1),(0,1)\}$ EKS ise:

1. $\left[\mu_{\mathrm{H}} \mathrm{SP}_{0}{ }^{\mathrm{D}}+\left(1-\mu_{\mathrm{H}}\right)\left(s \mathrm{P}^{\mathrm{Z}}+\mathrm{B}_{\mathrm{D}}\right)-\mathrm{c}\right]>\mathrm{rP}_{0}$

2. $\left[\mu_{\mathrm{H}} \mathrm{SP}_{0}{ }^{\mathrm{D}}+\left(1-\mu_{\mathrm{H}}\right)\left(\mathrm{sP}^{\mathrm{Z}}+\mathrm{B}_{\mathrm{D}}\right)-\mathrm{c}\right]<\mathrm{rP}_{0}$

3. $\delta_{\mathrm{G}}\left[\mathrm{rP}^{\mathrm{G}}-\mathrm{B}_{\mathrm{K}}\right]+\left(1-\delta_{\mathrm{G}}\right) \mathrm{sP}_{0}<0$.

Vaka 6. $\mathrm{p}^{*}=\{(0,1),(1,0),(0,1)\}$ EKS ise:

1. $\left[\mu_{H} s P_{0}^{D}+\left(1-\mu_{H}\right)\left(s P^{G}+B_{D}\right)-c\right]<r P_{0}$

2. $\left[\mu_{H} s P_{0}{ }^{D}+\left(1-\mu_{H}\right)\left(s P^{Z}+B_{D}\right)-c\right]>r P_{0}$

3. $\delta_{\mathrm{G}} \mathrm{SP}_{0}+\left(1-\delta_{\mathrm{G}}\right)\left[\mathrm{rP}^{Z}-\mathrm{B}_{\mathrm{K}}\right]<0$.

Vaka 7. $\mathrm{p}^{*}=\{(0,1),(0,1),(1,0)\}$ EKS ise: 
1. $\left[\mu_{\mathrm{H}} \mathrm{rP}_{0}{ }^{\mathrm{D}}+\left(1-\mu_{\mathrm{H}}\right)\left(\mathrm{rP}^{\mathrm{G}}-\mathrm{B}_{\mathrm{D}}\right)-\gamma\right]>\left[s \mathrm{P}^{\mathrm{G}}+\mathrm{B}_{\mathrm{K}}-\mathrm{c}\right]$

2. $\left[\mu_{\mathrm{H}} \mathrm{rP}_{0}{ }^{\mathrm{D}}+\left(1-\mu_{\mathrm{H}}\right)\left(\mathrm{rP}^{\mathrm{Z}}-\mathrm{B}_{\mathrm{D}}\right)-\gamma\right]>\left[\mathrm{sP}^{\mathrm{Z}}+\mathrm{B}_{\mathrm{K}}-\mathrm{c}\right]$

3. $\delta_{\mathrm{G}} \mathrm{s} \mathrm{P}_{0}+\left(1-\delta_{\mathrm{G}}\right) \mathrm{sP}_{0}>0$.

Vaka $8 \cdot \mathrm{p}^{*}=\{(0,1),(0,1),(0,1)\}$ EKS ise:

1. $\left[\mu_{H} s P_{0}^{D}+\left(1-\mu_{H}\right)\left(s P^{G}+B_{D}\right)-c\right]<\mathrm{rP}_{0}$

2. $\left[\mu_{H} s P_{0}{ }^{D}+\left(1-\mu_{H}\right)\left(s P^{Z}+B_{D}\right)-c\right]<\mathrm{rP}_{0}$

3. $\delta_{\mathrm{G}} \mathrm{SP}_{0}+\left(1-\delta_{\mathrm{G}}\right) \mathrm{sP}_{0}<0$.

Vaka $1,2,7$ ve 8 Mason ve diğerlerinin (2003) birleştiren dengelerini (pooling equilibria); 3, 4, 5 ve 6 ise ayrıştıran dengelerini (separating equilibria) çağnışırmaktadır. Ancak burada her vakanın evrimsel kararlı durum ya da replikatör dinamiklerinden sağ çıkabilen durum olması için gerekli olan kazanç yapılan da açık ve net olarak verilmektedir. Aynca her vakanın 3 numaralı koşuluna bakulacak olursa, hangi dengenin gerçekleşeceğinin oyuncu kümesindeki güçlü ve zayıf yatınmcıların oranlarına bağlı olduğu da anlaşılmaktadır.

Eğer oyuncular tam bilgiye sahip olsalardı, güçlü yatınmcılann hepsinin zorla elde etmeyi, zayıf yatırımcılann hepsinin pasif kaldığı ve de hedef yönetimlerin hepsinin işbirliği yaptığı tek bir denge bulunacaktı. Evrimsel oyunun olası kararl durumlarından Vaka 5 bu dengeyi de kapsamaktadır. Denklem 3'te bu dengenin kararlı olması için popülasyonda yer alması gereken güçlü ve zayıf yatırımcı oranının büyüklügüu yer almaktadır.

$$
\frac{\delta_{\mathrm{G}}}{1-\delta_{\mathrm{G}}}<\frac{\mathrm{sP}_{0}}{\mathrm{rP}^{\mathrm{G}}-\mathrm{B}_{\mathrm{K}}}
$$

Denklem 3'teki eşitsizliğe göre bu oran, güçlü yatırımcının pasif kalmaktan elde edeceği kazancın, yönetimin güçlü yatınmcı el koyduktan sonraki getirisine oranından küçük olmalıdır. Diğer olası sonuçların koşullarından, onların denge olabilmesi için gerekli oranlar da benzer şekilde hesaplanabilir.

\section{Sonuç ve Değerlendirmeler}

Mason ve diğerlerinin (2003) modellerinde ortaya koyduğu üç ayn ve oldukça geniş denge kümeleri vardır: birleştiren dengeler kümesi (yatınmcının tipinin ayırt edilemediği dengeler), aynıştıran dengeler kümesi (yatınmcı tipinin ayırt edildiği dengeler) ve yarı ayrıştınlabilen dengeler kümesi. Yazarlara göre üçüncü küme, yani tüm güçlü yatırımcıların ve bazı zayıf yatırımcıların zorla elde etmeyi seçtikleri ve de hedef yönetimlerin bazılarının direnip bazılannın 
işbirliği yaptığı dengeler, ampirik ve anekdot kanıtlara en uygun olan görünmektedir. Oysa bu makalede aynı etap oyununu evrimsel oyun olarak modelleyip evrimsel kararlı stratejileri bulduğumuzda, her yatınmcı tipi için agresif ve pasif stratejilerin denge stratejisi olduğu durumlar söz konusu olsa $\mathrm{da}$, hedef yönetim için aynı kararlı durum içinde farklı davranışlarda bulunulan bir denge görünmemektedir. Evrimsel oyunun tüm olası dengelerinde hedef yönetimlerin tamamı tek bir stratejiyi oynamaktadır (yani $p_{3}=0$ ya da $p_{3}=1$ ). Ayrıca Mason ve diğerlerinin (2003) bulduklan açık uçlu denge kümeleri yerine bu oyunda sadece sekiz olası denge bulunmakta ve her olası dengenin gerçekleşebilmesi için gereken kazanç yapıları belirtilmektedir. Böylece popülasyodaki yatınımcı tiplerinin olasılık dağılımı bilindiği zaman hangi durumun oyunun tek dengesi olacağını bulmak mümkün olmaktadır. Bu denge, geleneksel oyun teorisi denge kavramlanını aksine, oyuncularn rasyonel olduğu ya da rasyonelliğin ortak bilgi olduğu varsayımlarına dayanmamaktadır.

\section{Kaynakça}

CYERT, R.M / KANG, S-H. / KUMAR, P. (2002), "Corporate Governance, Takeovers, and TopManagement Compensation: Theory and Evidence," Management Science, 48/4: 453 469.

DAHYA, J.B. / MCCONNELL, J.J. (2005), “Outside Directors and Corporate Board Decisions," Journal of Corporate Finance, 11(1/2): 37-60.

GANDOLFO, G. (1996), Economic Dynamics (Berlin: Heidelberg; New York: Springer).

GARDNER, R. / MORRIS, M. (1991), "The Evolutionary Stability of Bluffing in a Class of Extensive Form Games," SELTEN, R. (Der.), Game Equilibrium Models I: Evolution and Game Dynamics (Berlin-Heidelberg: Springer-Verlag): 182-194.

HERMALIN, B.E. / WEISBACK, M.S. (2003), "Board of Directors as an Endogeneously Determined Institution: A Survey of Economic Literature," University of California, Berkeley Working Paper Series (FRBNY Economic Policy Review).

HU, X. / SHAPLEY, L.S. (2003), ^"On Authority Distributions in Organizations: Controls, "Games and Economic Behavior, 45: 153-170.

MASON, C.F. / GOTTESMAN, A.A. / PREVOST, A.K. (2003), "Shareholder Intervention, Managerial Resistance, and Corporate Control: A Nash Equilibrium Approach," The Quarterly Review of Economics and Finance, 43: 466-482.

NOE, T.H. / PI, L. (2000), "Learning Dynamics, Genetic Algorithms, and Corporate Takeovers," Journal of Economic Dynamics and Control, 24: 189-217.

SAMUELSON, L. (1997), Evolutionary Games and Equilibrium Selection (Cambridge, Massachusetts: The MIT Press).

SCHLAG, K. H. (1997), "Why Imitate, and if so, How? A Bounded Rationality Approach to Multiarmed Bandits," Journal of Economic Theory, 78: 127-159.

SELTEN, R. (1980), "A Note on Evolutionarily Stable Strategies in Asymmetric Animal Conflicts," Journal of Theoretical Biology, 84: 93-101. 
166. Ankara Üniversitesi SBF Dergisi • 62-2

SOYTAŞ, U. (2002), "Evrimsel Oyun Teorisi Üzerine Bir Not," Abant lzzet Baysal Üniversitesi Sosyal Bilimler Enstitüsü Dergisi, 5: 198-206.

SOYTAS, U. / BECKER, K.G. (2003), "Is Limit Pricing Evolutionarily Stable?," Journal of Evolutionary Economics, 13/3: 281-288.

SOYTAS, U. (2005), "The Role of Fixed Costs in an Evolutionary Entry Game with Bertrand Players," Hacettepe IIBF Dergisi, 23/2: 207-219.

TAYLOR, P. / JONKER, L. (1978), "Evolutionarily Stable Strategies and Game Dynamics," Mathematical Biosciences, 40: 145-156. 